\title{
Analysis of Error in the Solution of the 2-D Diffusion Equation by Finite Element Methods
}

\author{
L.F.M. MOURA ${ }^{1}$, E.C. ROMÃ̃ ${ }^{2}$, UNICAMP, Campinas, SP, Brasil. \\ J.B.C. SILVA ${ }^{3}$ FEIS, UNESP, Ilha Solteira, SP, Brasil.
}

\begin{abstract}
This work presents a numerical solution of the two-dimensional diffusion equation in comparison with the analytical solution. The norms $L_{2}$ and $L_{\infty}$ of the error are evaluated for two variants of the finite element method: the Galerkin Finite Element Method (GFEM) and the Least-Squares Finite Element Method (LSFEM). Two applications are presented and discussed.
\end{abstract}

Keywords. GFEM, LSFEM, Diffusion Equation.

\section{Introduction}

In many works the GFEM was applied to diffusion dominant problems yielding good results. In [4], GFEM was applied to solve nonlinear electro-hydrodynamic problems with the diffusive term stronger than the convective term.

In [5], a study of the computational efficiency of two numerical methods based on the Finite Difference/Galerkin Technique: Reduced Galerkin and Pseudo-Spectral was done for the steady state convection problem of Rayleigh-Benard. The author, after presenting the formulation of both methods, shows a numerical test that demonstrates graphically that the Pseudo-Spectral Method use a larger number of iterations than the Reduced Galerkin Method for the convergence of the solution.

In this work it is intended to compare results through the numerical simulation of diffusion problems by the GFEM with the LSFEM. One of the main objectives is the evaluation of the norms $L_{2}$ and $L_{\infty}$ for both GFEM and LSFEM and see what method may be more efficient considering the refine of the meshes of triangles and quadrilateral with linear or quadratic finite elements.

\section{Model Equation}

The partial differential equation that models the two dimensional diffusive phenomenon is a particular case of the field problem equation and it is defined in a

\footnotetext{
${ }^{1}$ felipe@fem.unicamp.br.

2estaner23@yahoo.com.br.

3jbcampos@dem.feis.unesp.br.
} 
domain $\Omega \subset \Re^{2}$, where $\Omega$ is a limited and closed. In the case of heat transfer the field of interest is the temperature field and the governing equation is of the form:

$$
\frac{\partial}{\partial x}\left(k \frac{\partial u}{\partial x}\right)+\frac{\partial}{\partial y}\left(k \frac{\partial u}{\partial y}\right)+f=0
$$

where $k$ is a positive constant; $u=u(x, y)$ is the variable, and $f=f(x, y)$ is a source term, with $x, y \in \Re$. The boundary conditions are of first and second kinds.

\section{Galerkin Finite Element Method}

For the application of this approach, it is necessary to define a variational formulation of the general problem described by the Equation (2.1). The variational formulation consists of finding $u^{e} \in V^{e}$ (where $V^{e} \in C^{2}(\Omega)$ ) such that

$$
\int_{\Omega^{e}}\left(\frac{\partial}{\partial x}\left(k \frac{\partial u}{\partial x}\right)+\frac{\partial}{\partial y}\left(k \frac{\partial u}{\partial y}\right)+f\right) v_{i}^{e} d x=0
$$

$\forall v_{i}^{e} \in V^{e}, i=1,2, \ldots, N_{\text {nodes }} . v_{i}^{e}$ are the weight functions resultant of the Weighted Residual Method definition.

For the spatial discretization, the following interpolation is used for the variable $u$ inside a finite element,

$$
u \cong u^{e}=\sum_{j=1}^{N_{\text {nodes }}} N_{i}(x, y) u_{i}^{e}
$$

where $N_{\text {nodes }}$ is the number of nodes inside each finite element. After substitution of Equation (3.2) in the Equation (3.1) and integration by parts as described in [3], with $v_{i}^{e}=N_{i}, i=1,2, \ldots, N_{\text {nodes }}$, one:

$$
[K]\left\{u^{e}\right\}=\{F\}
$$

In a local coordinates system $(\xi, \eta)$ in the elements he coefficients of the matrix $[K]$ and the vector $F$ are:

$$
\begin{gathered}
K_{i j}=\int_{\bar{\Omega}^{e}}\left[-\frac{k}{\operatorname{det}(J)^{2}} \Phi_{1} \Phi_{2}-\frac{k}{\operatorname{det}(J)^{2}} \Phi_{3} \Phi_{4}\right] \operatorname{det}(J) d \bar{\Omega} \\
F_{i}=-\int_{\bar{\Omega}^{e}} f N_{i} \operatorname{det}(J) d \bar{\Omega}+(\text { Boundary Term }),
\end{gathered}
$$

where,

$$
\begin{aligned}
& \Phi_{1}=\frac{\partial y}{\partial \eta} \frac{\partial N_{i}}{\partial \xi}-\frac{\partial y}{\partial \xi} \frac{\partial N_{i}}{\partial \eta} \\
& \Phi_{2}=\frac{\partial y}{\partial \eta} \frac{\partial N_{j}}{\partial \xi}-\frac{\partial y}{\partial \xi} \frac{\partial N_{j}}{\partial \eta}
\end{aligned}
$$




$$
\begin{gathered}
\Phi_{3}=-\frac{\partial x}{\partial \eta} \frac{\partial N_{i}}{\partial \xi}+\frac{\partial x}{\partial \xi} \frac{\partial N_{i}}{\partial \eta} \\
\Phi_{4}=-\frac{\partial x}{\partial \eta} \frac{\partial N_{j}}{\partial \xi}+\frac{\partial x}{\partial \xi} \frac{\partial N_{j}}{\partial \eta}
\end{gathered}
$$

with $i, j=1, \ldots, N_{\text {nodes }}$.

Further details of this formulation can be found in [8], chapter 8 .

\section{Least-Square Finite Element Method}

Initially for the application of the LSFEM for two dimensional problems two auxiliary variables are defined generating a system of three partial differential equations with three unknowns, as follows,

$$
-\frac{\partial q_{x}}{\partial x}-\frac{\partial q_{y}}{\partial y}+f=0 \quad q_{x}+k \frac{\partial u}{\partial x}=0 \quad q_{y}+k \frac{\partial u}{\partial y}=0 .
$$

This artifice decreases the order of the problem and, consequently, the order of the involved weight and interpolations functions, [10]. Now, we done the interpolation in each element to the $u^{e}, q_{x}^{e}$ e $q_{y}^{e}$ functions, in the following way:

$$
\begin{gathered}
u \cong u^{e}=\sum_{i=1}^{N_{\text {nodes }}} N_{i} u_{i}^{e} \\
q_{x} \cong q_{x}^{e}=\sum_{i=1}^{N_{\text {nodes }}} N_{i} q_{x i}^{e} \\
q_{y} \cong q_{y}^{e}=\sum_{i=1}^{N_{\text {nodes }}} N_{i} q_{y i}^{e}
\end{gathered}
$$

After the definition of the spatial approximations, the following residuals can be defined to Equations (4.1), [6]:

$$
\begin{gathered}
R_{1}(x, y)=-\frac{\partial q_{x}}{\partial x}-\frac{\partial q_{y}}{\partial y}+f \\
R_{2}(x, y)=q_{x}+k \frac{\partial u}{\partial x} \\
R_{3}(x, y)=q_{y}+k \frac{\partial u}{\partial y}
\end{gathered}
$$

In this way, the residuals are functions of the nodal variables $u_{i}^{e}, q_{x i}^{e}$ and $q_{y i}^{e}$. The LSFEM consists in the definition and minimization of the following quadratic functional [2], 


$$
I\left(R_{1}, R_{2}, R_{3}\right)=\int_{\Omega^{e}} R_{1}^{2}(x, y) d \Omega+\int_{\Omega^{e}} R_{2}^{2}(x, y) d \Omega+\int_{\Omega^{e}} R_{3}^{2}(x, y) d \Omega
$$

for all $u \in V=\left\{u \in H^{1}(\Omega)\right\}$, where $H^{1}$ is the Hilbert space of order one.

The necessary condition for minimization of the functional in the Equation (4.8) is that its first variation be null. Then, we obtain

$$
\int_{\Omega^{e}}\left(\delta R_{1}\right) R_{1} d \Omega+\int_{\Omega^{e}}\left(\delta R_{2}\right) R_{2} d \Omega+\int_{\Omega^{e}}\left(\delta R_{3}\right) R_{3} d \Omega=0
$$

Where the first variations of the residuals are

$$
\begin{gathered}
\delta R_{1}=\frac{\partial R_{1}}{\partial u_{i}^{e}} \delta u_{i}^{e}+\frac{\partial R_{1}}{\partial q_{x i}^{e}} \delta q_{x i}^{e}+\frac{\partial R_{1}}{\partial q_{y i}^{e}} \delta q_{y i}^{e} \\
\delta R_{2}=\frac{\partial R_{2}}{\partial u_{i}^{e}} \delta u_{i}^{e}+\frac{\partial R_{2}}{\partial q_{x i}^{e}} \delta q_{x i}^{e}+\frac{\partial R_{2}}{\partial q_{y i}^{e}} \delta q_{y i}^{e} \\
\delta R_{3}=\frac{\partial R_{3}}{\partial u_{i}^{e}} \delta u_{i}^{e}+\frac{\partial R_{3}}{\partial q_{x i}^{e}} \delta q_{x i}^{e}+\frac{\partial R_{3}}{\partial q_{y i}^{e}} \delta q_{y i}^{e}
\end{gathered}
$$

The substitution of the equations (4.2-4.4) and (4.10-4.12) in Equation (4.9), yields in a local coordinates system $(\xi, \eta)$, where $-1 \leq \xi, \eta \leq 1[7]$, to the following matrix system of equations:

$$
\left[\begin{array}{ccc}
A & B & C \\
B^{T} & D & E \\
C^{T} & E^{T} & G
\end{array}\right]\left\{\begin{array}{l}
\left\{u^{e}\right\} \\
\left\{q_{x}^{e}\right\} \\
\left\{q_{y}^{e}\right\}
\end{array}\right\}=\left\{\begin{array}{l}
\left\{F_{1}\right\} \\
\left\{F_{2}\right\} \\
\left\{F_{3}\right\}
\end{array}\right\}
$$

where the coefficients of the matrices are defined as

$$
\begin{gathered}
A_{i j}=\int_{\bar{\Omega}^{e}}\left\{\frac{k^{2}}{\operatorname{det}(J)^{2}} \Phi_{1} \Phi_{2}+\frac{k^{2}}{\operatorname{det}(J)^{2}} \Phi_{3} \Phi_{4}\right\} \operatorname{det}(J) d \bar{\Omega} \\
B_{i j}=\int_{\bar{\Omega}^{e}}\left\{k \Phi_{1} N_{j}\right\} d \bar{\Omega} \\
C_{i j}=\int_{\bar{\Omega}^{e}}\left\{k \Phi_{3} N_{j}\right\} d \bar{\Omega} \\
D_{i j}=\int_{\bar{\Omega}^{e}}\left\{\frac{1}{\operatorname{det}(J)^{2}} \Phi_{1} \Phi_{2}+N_{i} N_{j}\right\} \operatorname{det}(J) d \bar{\Omega} \\
E_{i j}=\int_{\bar{\Omega}^{e}}\left\{\frac{1}{\operatorname{det}(J)} \Phi_{1} \Phi_{4}\right\} d \bar{\Omega} \\
G_{i j}=\int_{\bar{\Omega}^{e}}\left\{\frac{1}{\operatorname{det}(J)^{2}} \Phi_{3} \Phi_{4}+N_{i} N_{j}\right\} \operatorname{det}(J) d \bar{\Omega}
\end{gathered}
$$




$$
F_{1 i}=0, F_{2 i}=\int_{\bar{\Omega}^{e}}\left\{\Phi_{1} f\right\} d \bar{\Omega}, F_{3 i}=\int_{\bar{\Omega}^{e}}\left\{\Phi_{3} f\right\} d \bar{\Omega}
$$

with $i, j=1, \ldots, N_{\text {nodes }}$ and $\Phi_{1}, \Phi_{2}, \Phi_{3}$ and $\Phi_{4}$ defined in Equations (3.6-3.9). Further details of this formulation can be found in [8].

\section{Norms $L_{2}$ and $L_{\infty}$}

In this work will be evaluated the norms $L_{2}$ and $L_{\infty}$ of the error in the numerical solutions by the GFEM and LSFEM compared with the analytical solution. Consider $u$ as being the numerical solution and $u_{a}$ the analytical solution of the Equation (2.1), the norms can be defined as: $L_{2 N o r m}=\sqrt{\sum_{i=1}^{N_{\text {nodes }}}\left|u(i)-u_{a}(i)\right|}$ and $L_{\infty \text { Norm }}=\max \left|u(i)-u_{a}(i)\right|, i=1, \ldots, N_{\text {nodes }}$.

\section{Numerical Applications}

The integrations are done numerically by the Gauss-Legendre Quadrature. The Gauss-Legendre Quadrature requests that the integrals be evaluated on the interval $[-1,1] \times[-1,1]$, i. e., it requests a transformation of the problem of the global coordinates $x$ and $y$ to a local coordinates $\xi$ and $\eta$, as already mentioned in the item (3.) and (4.). For the solution of the matrix system the Frontal Method, [9] pp 120-153, cap. 6 has been applied and for the mesh generation the Ansys (1994) was used.

In the following sections two applications, in which the main objective is to evaluate the norms $L_{2}$ and $L_{\infty}$ of the error of the numerical solutions against the analytical solution will be presented. The analytical solutions presented can be found in [1].

\subsection{First Application: Pure Diffusion with boundary condi- tions of the first kind}

In this application a purely steady state diffusive case with all the boundary conditions of the first kind, as shown in Figure 1, has been analyzed. The governing Equation $(2.1)$ reduces to $\frac{\partial^{2} u(x, y)}{\partial x^{2}}+\frac{\partial^{2} u(x, y)}{\partial y^{2}}=0$ The analytical solution to this case is of the form:

$$
u(x, y)=\frac{4}{\pi} \sum_{n=0}^{\infty} \frac{\sinh [(2 n+1) \pi(1-x)]}{\sin [(2 n+1) \pi]} \frac{\sin [(2 n+1) \pi y]}{2 n+1}
$$

so, the first derivatives of the variable $u$ is

$$
\frac{\partial u(x, y)}{\partial x}=-4 \sum_{n=0}^{\infty} \frac{\cosh [(2 n+1) \pi(1-x)]}{\sin [(2 n+1) \pi]} \sin [(2 n+1) \pi y]
$$




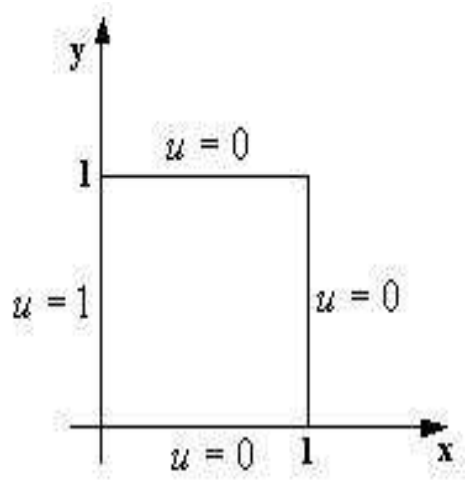

Figure 1: Cartesian Geometry with boundary conditions of the first kind.

$$
\frac{\partial u(x, y)}{\partial y}=4 \sum_{n=0}^{\infty} \frac{\sinh [(2 n+1) \pi(1-x)]}{\sin [(2 n+1) \pi]} \cos [(2 n+1) \pi y]
$$

In this work meshes of linear and quadratic quadrilateral and triangular elements have been used. The Figure 2 illustrates a mesh of 1600 quadrilateral elements of size $\left(h=2,5 \times 10^{-2}\right)$. The size $h=\Delta x \times \Delta y$ of the element was varied in the range of $10^{-1}$ to $2,5 \times 10^{-2}$. The quadratic quadrilateral element used was the serendipity element of eight nodes and the results from these meshes were better than the results from linear four nodes elements, as expected. In the present analysis of the norms

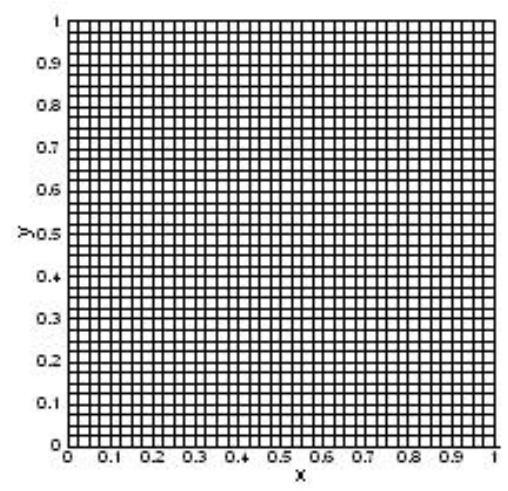

Figure 2: Mesh with 1600 quadrilateral elements.

$L_{2}$ and $L_{\infty}$ the numerical solutions by the GFEM and LSFEM to $u, \partial u / \partial x$ and $\partial u / \partial y$ solutions have been computed and are presented in the following figures.

Figure 3(a) illustrates the norm $L_{2}$ from the solution of $u$. It can be seen that with the eight noded element both to the GFEM and the LSFEM produced similar results, while with the linear element the GFEM presented better results than the LSFEM. 


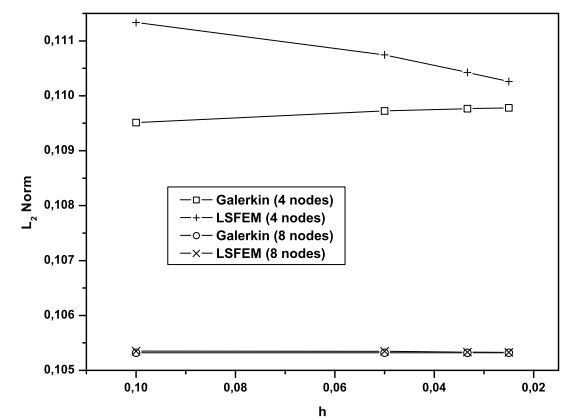

(a)

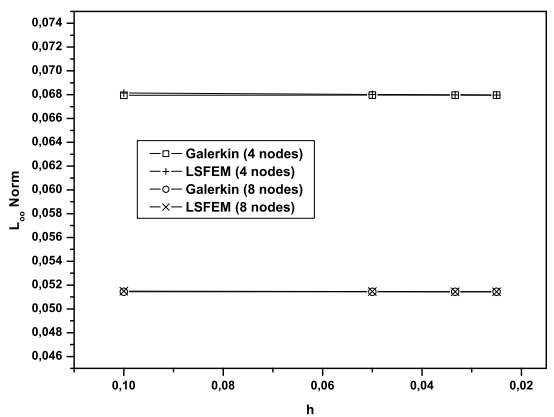

(b)

Figure 3: (a) Norm $L_{2}$ of error in numerical solution $u$ for meshes with quadrilateral elements with four and eight nodes. (b) Norm $L_{\infty}$ of error in numerical solution $u$ for meshes with quadrilateral elements with four and eight nodes.

Figure 3(b) shows the norm $L_{\infty}$ from the solution of $u$. It can be seen that in this case, the eight noded element both to the GFEM and LSFEM produced similar results, better than the similar results from the linear elements.

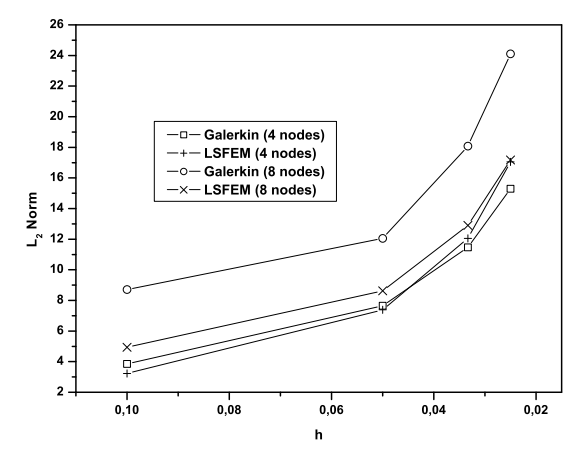

(a)

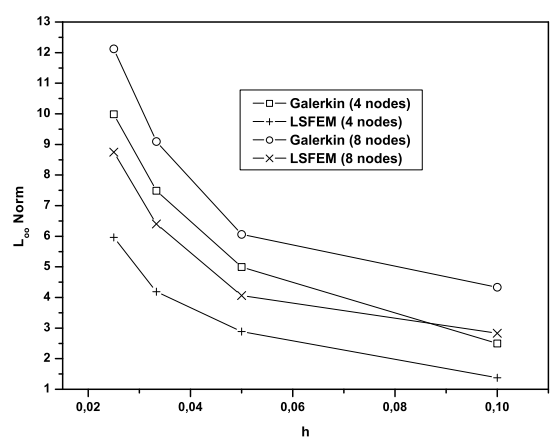

(b)

Figure 4: (a) Norm $L_{2}$ of error in numerical solution $\partial u / \partial x$ for meshes with quadrilateral elements with four and eight nodes. (b)Norm $L_{\infty}$ of error in numerical solution $\partial u / \partial x$ for meshes with quadrilateral elements with four and eight nodes.

The Figures 4 and 5 show the norms in the comparisons of the solutions of the first derivatives, $\partial u / \partial x$ and $\partial u / \partial y$. In this case a strange behavior occurred: when the meshes were refined the results became worst, mainly, in the regions of the corners $(x, y)=(0,0)$ and $(x, y)=(0,1)$ where large gradients are present. These 
corners are singular points and maybe it explains the bad results.

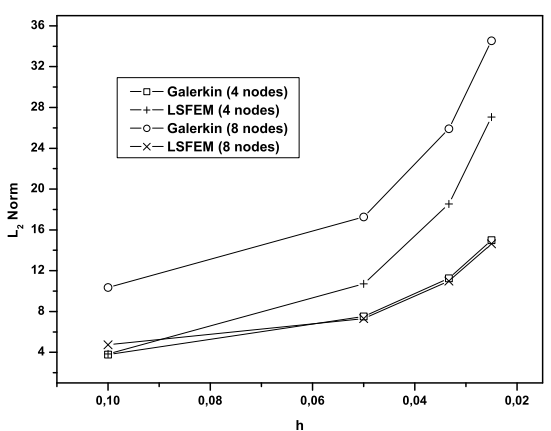

(a)

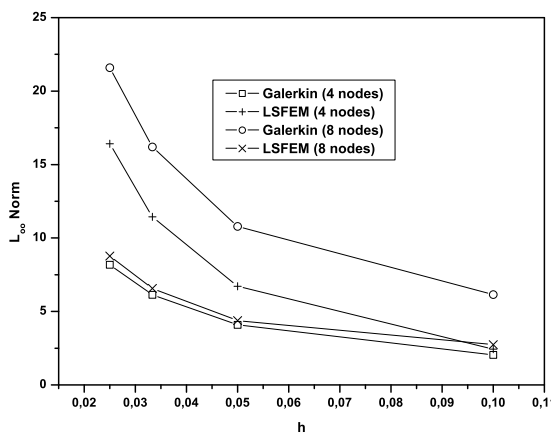

(b)

Figure 5: (a) Norm $L_{2}$ of error in numerical solution $\partial u / \partial y$ for meshes with quadrilateral elements with four and eight nodes. (b)Norm $L_{\infty}$ of error in numerical solution $\partial u / \partial y$ for meshes with quadrilateral elements with four and eight nodes.

As observed in the case of the solution $u$, quadratic elements yield to better results than the linear elements, also, in this case of the derivatives.

\subsection{Second Application: Pure Diffusion with boundary con- ditions of first and second kind.}

In this case a purely diffusive problem with source term and boundary conditions of first and second kind has been analyzed. Now at the contours $x=0$ and $y=0$ the fluxes are specified as shown in Figure 6. In this case another difference is that the meshes are of linear and quadratic triangular elements of size $h$ varying from $2,82 \times 10^{-1}$ to $3,54 \times 10^{-2}$. Figure 7 illustrates a mesh of 3200 triangular elements. The norms $L_{2}$ and $L_{\infty}$ were computed and are presented in Figures 8(a) to 10(a). The governing equation in this case is:

$$
\frac{\partial^{2} u(x, y)}{\partial x^{2}}+\frac{\partial^{2} u(x, y)}{\partial y^{2}}+5=0
$$

The analytical solution of this application is of the form:

$$
u(x, y)=5 \times\left\{\frac{1}{2}\left(1-x^{2}\right)-2 \sum_{n=0}^{\infty} \frac{(-1)^{n}}{\lambda_{n}^{3}} \frac{\cosh \left(\lambda_{n} y\right)}{\cosh \left(\lambda_{n}\right)} \cos \left(\lambda_{n} x\right)\right\},
$$

so, the first derivatives are evaluated as

$$
\frac{\partial u(x, y)}{\partial x}=5 \times\left\{-x+2 \sum_{n=0}^{\infty} \frac{(-1)^{n}}{\lambda_{n}^{2}} \frac{\cosh \left(\lambda_{n} y\right)}{\cosh \left(\lambda_{n}\right)} \sin \left(\lambda_{n} x\right)\right\},
$$




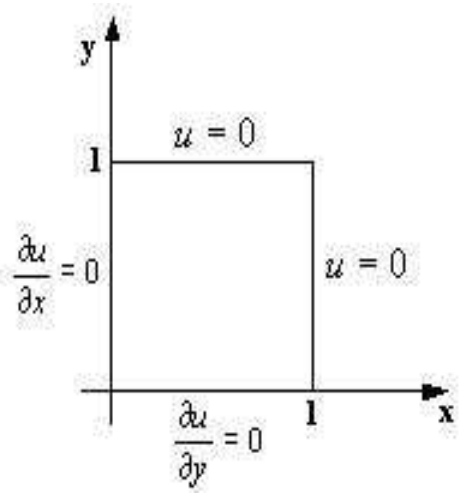

Figure 6: Cartesian Geometry with boundary conditions of first and second type.

$$
\frac{\partial u(x, y)}{\partial y}=-10 \sum_{n=0}^{\infty} \frac{(-1)^{n}}{\lambda_{n}^{2}} \frac{\sinh \left(\lambda_{n} y\right)}{\cosh \left(\lambda_{n}\right)} \cos \left(\lambda_{n} x\right)
$$

where $\lambda_{n}=(2 n+1) \pi / 2$ and $n=0,1,2, \ldots$

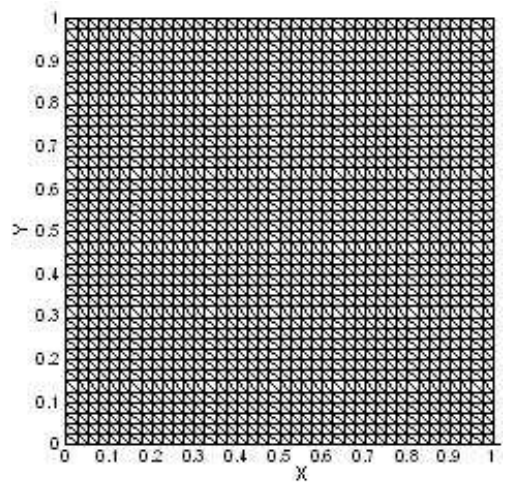

Figure 7: Mesh with 3200 triangular elements.

The norms $L_{2}$ and $L_{\infty}$ of the error in the numerical solutions from the GFEM and LSFEM to the variable $u$ and first derivatives $\partial u / \partial x$ and $\partial u / \partial y$ with meshes of three and six noded triangular elements are presented in next figures.

In Figures 8 (a) and (b) it can be observed that with the refine of the meshes better results are obtained. Quadratic six node triangular elements produced better results than the results from linear triangular elements. Both the GFEM and the LSFEM presented similar results for the case of quadratic element, while to the case of linear elements the GFEM presented better results than the LSFEM.

In the case of this application, contrary to the first application, the derivatives 


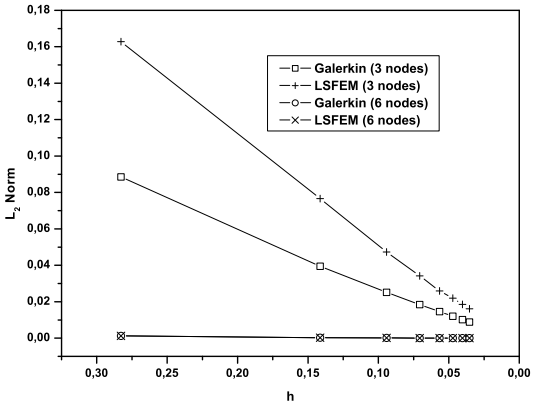

(a)

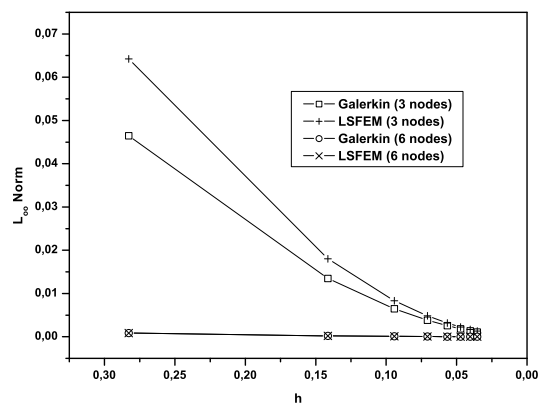

(b)

Figure 8: (a) Norm $L_{2}$ of error in numerical solution $u$ for meshes with triangular elements with three and six nodes. (b) Norm $L_{\infty}$ error in numerical solution $u$ for meshes with triangular elements with three and six nodes.

$\partial u / \partial x$ and $\partial u / \partial y$ were evaluated without any strange behavior. And the solutions of the derivatives are enhanced with the refine of the meshes.

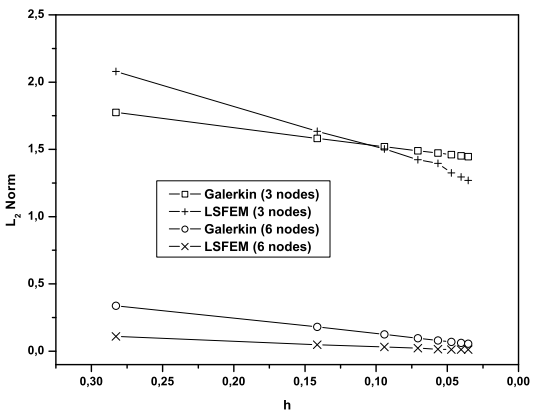

(a)

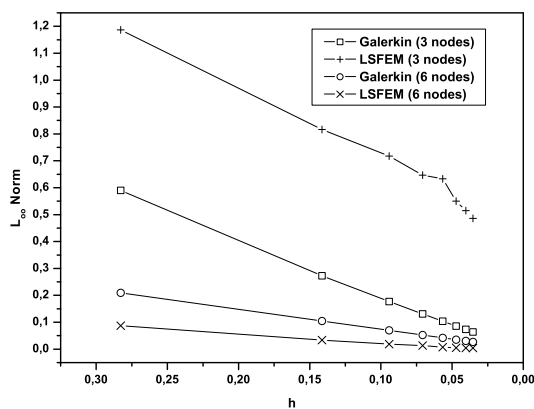

(b)

Figure 9: (a) Norm $L_{2}$ of error in numerical solution $u$ for meshes with triangular elements with three and six nodes. (b) Norm $L_{\infty}$ error in numerical solution $\partial u / \partial x$ for meshes with triangular elements with three and six nodes

From analysis of Figures 9 to 10 it can be noticed that the LSFEM presented the best results to the norms of the errors in the derivatives when quadratic elements were used. When linear elements were used the GFEM presented the best results compared with the LSFEM. 


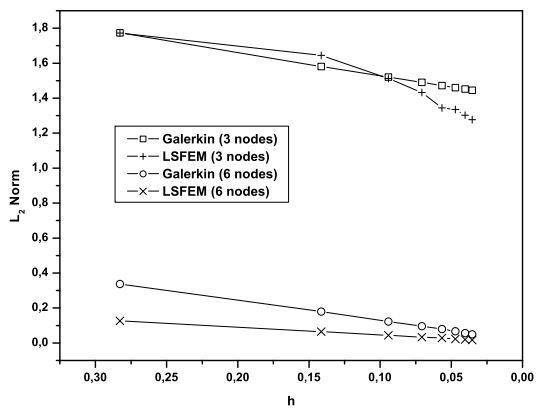

(a)

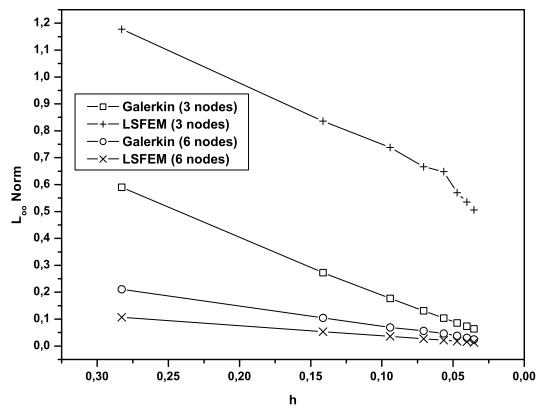

(b)

Figure 10: (a) Norm $L_{2}$ error in numerical solution $\partial u / \partial y$ for meshes with triangular elements with three and six nodes. (b) Norm $L_{\infty}$ error in numerical solution $\partial u / \partial y$ for meshes with triangular elements with three and six nodes.

\section{Conclusion}

Initially, it is interesting to mention the fact, positive, of simultaneous computation of the fluxes in the LSFEM. Because the use of auxiliary variables, the fluxes, the numbers of degrees of freedom per node increases, consequently, increase the computational cost. However, this disadvantage is a little alleviated if we consider that the matrix of the coefficients, although larger, is symmetrical, facilitating the storage of just approximately half of its elements. Another advantage of the LSFEM is that the resulting matrix is also positive defined and, so, spurious solutions are prevented. This characteristic of the matrix in LSFEM facilitate the usage of efficient methods of solutions such as preconditioned conjugate gradient method. When all boundary conditions are of first kind the LSFEM has its efficiency decreased, in concerning of heat fluxes calculation, maybe because no boundary condition in this case be specified to the fluxes. However, with the refine of the meshes better results may be obtained with the increase of the cost computational. For more gross meshes the GFEM present better results than the LSFEM. For purely diffusive problems and when there is not need to calculate the fluxes the best method is the GFEM. When there is the need to calculate the fluxes the best method is the LSFEM, because the GFEM requires a post processing to the calculation of the fluxes.

\section{Acknowledgment.}

The present work was supported by the National Council of Scientific Development and Technology - CNPq - Brasil.

Resumo. Neste trabalho apresenta-se a solução numérica via Método dos Elementos Finitos da equação de difusão bidimensional linear sendo comparada com a solução analítica. Para tal análise serão utilizadas as normas $L_{2}$ e $L_{\infty}$ do erro na 
utilização de duas variantes do Método dos Elementos Finitos: Galerkin e Minimos Quadrados. Duas aplicações são apresentadas e discutidas.

Galerkin, Mínimos Quadrados, Equação da Difusão.

\section{References}

[1] V.S. Arpaci, "Conduction Heat Transfer", Addison-Wesley Publishing Company, 1966.

[2] T.J. Chung, "Finite Element Analysis in Fluid Dynamics", McGraw-Hill, 1978.

[3] G. Dhatt, G. Touzot, "The Finite Element Method", John Wiley \& Sons, 1984.

[4] J.Q. Feng, Application of Galerkin finite-element computations in studying electro hydrodynamic problems, Journal of Electrostatic, 51-52 (2001), 590596.

[5] L.E. Howle, A comparison of the reduced Galerkin and pseudo-spectral methods for simulation of steady Rayleigh-Benard convection, International Journal Heat Mass Transfer, 39, No. 12 (1996), 2401-2407.

[6] B.N. Jiang, "The Least-Squares Finite Element Method: Theory and Applications in Computational Fluid Dynamics and Electromagnetic", Springer, 1998.

[7] J.N. Reddy, "An Introduction to the Finite Element Method", Second Edition, McGraw-Hill, 1993.

[8] E.C. Romão, "Variantes do Método dos Elementos Finitos para Solução de Fenômenos Convectivo-Difusivos Bidimensionais", Dissertação de Mestrado, DEM/FEIS, UNESP, Ilha Solteira, SP, 2004.

[9] C. Taylor,T.G. Hughes, "Finite Element Programming of the Navier-Stokes Equations", Pineridge Press Limited, Swansea, 1981.

[10] D. Winterscheidt, K.S. Surana, P-version least-squares finite element formulation for convection-diffusion problems, International Journal for Numerical Methods in Engineering, 36 (1993), 111-133. 